\section{Research Square \\ Preprints are preliminary reports that have not undergone peer review. \\ They should not be considered conclusive, used to inform clinical practice, or referenced by the media as validated information.}

\title{
Protocol for a systematic review on classification, outcomes and follow-up of patients undergoing reconstructive surgery after Noma disease-
}

Sophie Speiser ( $\nabla$ s.speiser1@gmail.com )

University College London Division of Surgery and Interventional Science https://orcid.org/0000-0001-7099-4395

Benjamin Langridge

University College London Division of Surgery and Interventional Science

Moira Melina Birkl

Karl Landsteiner Privatuniversitat fur Gesundheitswissenschaften

Harald Kubiena

Medical University of Vienna: Medizinische Universitat Wien

Will Rodgers

Great Ormond St Hosp Children: Great Ormond Street Hospital For Children NHS Foundation Trust

\section{Protocol}

Keywords: "Noma”, "cancrum oris", "ulcerative necrotizing gingivitis", "facial reconstructive surgery”, "global developmental surgery", "mission surgery”, "classification", "outcomes", "complications", "trismus", "follow-up"

Posted Date: October 13th, 2020

DOI: https://doi.org/10.21203/rs.3.rs-89911/v1

License: @ (i) This work is licensed under a Creative Commons Attribution 4.0 International License. Read Full License 


\section{Abstract}

Background: NOMA is a significant yet neglected disease which affects children in some of the least developed countries in the world. Surgical reconstructive missions for patients affected by NOMA are undertaken regularly by surgeons from more developed countries. However, the long-term benefit and safety of these missions have recently been criticized due to a perceived lack of measurable outcomes and appropriate follow-up. We are conducting a systematic review to critically evaluate the classification systems, outcome measurement tools, and follow-up periods reported in the literature for NOMA disease.

Methods: This systematic review is undertaken following the Preferred Reporting Items for Systematic Reviews and Meta-analyses ("PRISMA") guidelines. The databases MEDLINE, Embase and Web of Science are searched from infinity to $24^{\text {th }}$ of April 2019. All original publications of studies undertaken on reconstructive surgery for NOMA disease, reporting data on outcome after surgery, follow-up time and complications are eligible for inclusion. The primary outcome of interest are the outcome measurement methods used for assessing successful reconstructive surgery after NOMA disease. Other outcomes we are assessing are methods for classification of disease severity and time to last follow-up. The quality of evidence for each summary outcome measure is assessed using the MINORS criteria.

Discussion: Given the precarious situations in the countries where mission surgeons often undertake reconstructive surgery, time pressure and the lack of infrastructure on site prevent them from being able to measure the effect of their surgical outcomes. Our goal is to express the key points of debate and make recommendations for how future surgical missions can be improved. We aim to do so by comparing the current best standards of practice Publishing this protocol will help future NOMA surgeons plan their missions in a standardised and reproducible way, striving towards a future of worldwide high-quality research.

Systematic review registrations: This protocol was submitted to PROSPERO on the 02.10 .2020 and is currently under assessment. (ID 181931)

\section{Background}

NOMA is a gangrenous ulcerating bacterial disease of multifactorial origin, mostly prevalent in sub-saharan Africa. Due to the high reported mortality rate of up to $85-90 \%$, and the frequent occurrence in extreme poverty regions, WHO has declared NOMA a public health problem in 1994. (1) Global incidence is reported as 30 000-140 000 new cases per year $(1,2)$, and there are currently 210000 NOMA survivors worldwide, according to latest estimations $(2)$.

Most cases of NOMA occur between the age of 2-7 years. Malnutrition, poor oral hygiene and poverty are known etiological factors leading to nutritionally acquired immune deficiency syndrome. $(3,4)$ Children with this syndrome present with lymphatic atrophy and malfunction of cellular immune response, accompanied by an increased vulnerability to infections (5). These preconditions lead to the uptake of pathogens through the gingival mucosa. (6, 7) After uptake of opportunistic pathogens, progression to NOMA disease occurs in several stages, starting with a simple gingivitis and ending in a stage of sequelae including trismus, feeding difficulties and mild to severe disfigurement. (1)

Once the gangrenous gingivitis phase starts, this disease does not confine itself to anatomical or tissue borders. It can destroy the oral mucous integriy, facial fat and soft tissue and invades into the bone of the maxilla and mandible, leading to severe functional deficits (8).

Without appropriate treatment, the majority of affected patients do not survive. They die due to sepsis, aggravation of malnutritional status and following dehydration. Many children die without ever receiving treatment or even diagnosis, due to lack of health care infrastructure, little knowledge and neglect of the disease by the parents.(2)

Purpose of this systematic review is to explore the surgical treatment strategies, patient follow-up and outcomes of surgery with emphasis on outcomes of NOMA surgery.

\section{We are examining the following questions:}

1. What are the baseline characteristics of patients undergoing reconstructive surgery for NOMA disease?

2. Which surgical procedures are undertaken to reconstruct the face after NOMA disease?

3. What are the methods/tools for measurement of outcomes for these surgical procedures?

4. Are the current procedures undertaken safe and beneficial for NOMA patients?

a. Safety is measured in amount and types of complications and follow-up length

b. Benefit is measured as stability of postoperative outcome at follow-up

\section{The main outcomes we are looking at are:}

1. Safety and benefit of reconstructive surgery after NOMA disease including number and types of complications and means of measurement of outcome benefit.

2. Long-term benefit of reconstructive surgery after NOMA disease in the long-term follow-up including means of measurement of outcome benefit.

\section{Other outcomes of interest are:}

3. Overview on the basic characteristics of the studies including number of patients that received surgery, gender, health status prior to surgery (weight, BMI, comorbidities) 
4. Overview on the disease characteristics of the patients including affected areas and functions of the face and the classification of these

5. Overview on type of surgical intervention received, including site of intervention, type of surgeon (local or mission surgeon), number of operations needed and hospital duration

\section{Methods}

A comprehensive, structured literature search of published articles is conducted. This is designed by the author S.S and performed in adherence to the Preferred Reporting Items for Systematic Reviews and Meta-analyses (PRISMA), the standardized PRISMA-P checklist can be found in the supplementary material (9).

\subsection{Data sources}

The literature search is performed using the electronic databases MEDLINE (PubMed), EMBASE and Web of Science. The keywords used in the search are selected from key papers and are described in tables below. Search strings 1 and 2 are combined using the Boolean term AND, then the limits are applied. Furthermore, a 'MeSH term' search is conducted, and the reference lists of included articles are reviewed for any additional articles.

\section{Search strategy for Embase.com (15.04.2020)}

/exp = EMtree keyword with explosion

$. t w=$ The Textword field in EMBASE includes Title (TI) and Abstract (AB).

Table 1- Search Strategy used in EMBASE

\begin{tabular}{|c|c|c|}
\hline Number & Search terms & Results \\
\hline$\# 1$ & 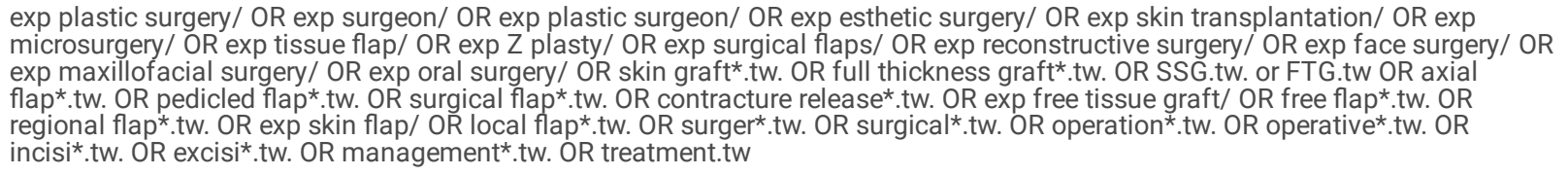 & 8557452 \\
\hline \#2 & $\begin{array}{l}\text { Exp Vincent stomatitis/ OR acute necrotizing ulcerative gingivitis.tw OR acute necrotising ulcerative gingivitis.tw OR acute } \\
\text { ulcerative gintivitis.tw OR noma.tw OR cancrum oris.tw }\end{array}$ & 1401 \\
\hline \#3 & \#1 AND \#2 & 639 \\
\hline
\end{tabular}

Limits: Publication prior to final search, April 15th, 2020

Source: Authors table

Search strategy for MEDLINE (PubMed) (15.04.2020)

/exp = MEDLINE keyword with explosion/ Mesh terms

$. t w=$ The Textword field in MEDLINE includes Title (TI) and Abstract (AB).

Table 2-Search Stratedy used in MEDLINE

\begin{tabular}{|c|c|c|}
\hline Number & Search terms & Results \\
\hline$\# 1$ & $\begin{array}{l}\text { exp Reconstructive Surgical Procedures/ OR exp Surgery, Plastic/ OR exp Tissue Transplantation/ OR exp Surgical Flaps/ OR exp } \\
\text { Skin Transplantation/ ORexp Free Tissue Flaps/ OR exp Microsurgery/ OR exp Surgery, Oral/ OR exp Surgical Procedures, } \\
\text { Operative/ OR exp "Oral and Maxillofacial Surgeons"/ OR exp Surgeons/ OR skin graft*.tw. OR full thickness graft*.tw. OR SSG.tw. } \\
\text { OR FTG.tw. OR axial flap }{ }^{\star} \text {.tw. OR pedicle flap*.tw. OR pedicled flap*.tw. OR surgical flap*.tw. OR contracture release*.tw. OR free } \\
\text { flap*.tw. OR regional flap*.tw. OR local flap*.tw. OR surger*.tw. OR surgical*.tw. OR operation*.tw. OR operative*.tw. OR incisi*.tw. } \\
\text { OR excisi*.tw. OR management*.tw. OR treatment*.tw }\end{array}$ & 8167413 \\
\hline \#2 & $\begin{array}{l}\text { exp Noma/ OR cancrum oris.tw. OR exp Gingivitis, Necrotizing Ulcerative/ OR acute necrotizing ulcerative gingivitis.tw. OR acute } \\
\text { necrotising ulcerative gingivitis.tw. }\end{array}$ & 1664 \\
\hline \#3 & \#1 AND \#2 & 568 \\
\hline
\end{tabular}

Source: Authors table

Search strategy for Clarivate Analytics/Web of Science (15.04.2020)

TOPIC $=$ words in title, abstract or keywords

ndexes=SCI-EXPANDED, SSCI, A\&HCl, CPCI-S, CPCI-SSH, BKCl-S, BKCI-SSH, ESCI, CCR-EXPANDED, IC Timespan=All years

Table 3- Search Strategy used in Web of Science 


\begin{tabular}{|c|c|c|}
\hline Number & Search terms & Results \\
\hline$\# 1$ & 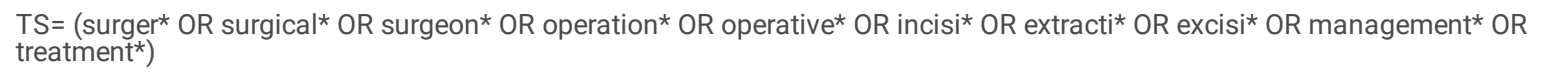 & $9,621,445$ \\
\hline \#2 & $\begin{array}{l}\text { TS }=(\text { reconstructi* OR cosmetic* OR esthetic* OR aesthetic* OR plastic OR corrective* OR oral* or *maxillofacial* OR facial OR } \\
\text { face* OR "head and neck" OR flap* OR skin* or micro*) }\end{array}$ & $8,095,337$ \\
\hline \#3 & $\begin{array}{l}\text { TS= (noma OR "cancrum oris" OR "necrotizing ulcerative gingivitis" OR "acute necrotizing ulcerative gingivitis" OR "acute } \\
\text { necrotising ulcerative gingivitis") }\end{array}$ & 3,515 \\
\hline \#4 & \#2 AND \#1 & $1,821,209$ \\
\hline \#5 & \#4 AND \#3 & 186 \\
\hline
\end{tabular}

Limits: Publication prior to final search, April 15th, 2020

The PRISMA flow chart is used to display the selection and inclusion/exclusion of articles in the screeining process, as seen below.

Source: Moher D, Shamseer L, Clarke M, Ghersi D, Liberati A, Petticrew M, et al. Preferred reporting items for systematic review and meta-analysis protocols (PRISMA-P) 2015 statement. Syst Rev. 2015;4:1.

\subsection{Study eligibility criteria}

Articles are eligible for inclusion if they provide primary empirical evidence of patient outcomes after NOMA surgery. The full inclusion and exclusion criteria are detailed in the table below. Study selection was independently assessed by 2 reviewers (S.S. and M.B.), any persisting disagreements were reviewed and adjudicated by a third reviewer (H.K.).

Table 5- Inclusion/exclusion criteria for study selection

Inclusion Criteria
1. Original publications
2. Human subjects
3. Case Series, Retrospective Data Analyses, Clinical Trials, Controlled Clinical Trials, Prospective Studies
Exclusion Criteria
1. Publication type does not match inclusion criteria (Review articles, Mixed methodology studies without subgroup data, Animal studies, Conference
Articles)
2. Conditions other than NOMA, Cancrum Oris, or Necrotizing Ulcerative Stomatitis not related to NOMA
3. The paper is about conditions located at sites other than the Maxillofacial and Head and Neck region
4. The paper is not about treatment
5. The paper is not about a surgical intervention (but included if terms such as reconstruction or rehabilitation, or flap or graft, are mentioned).
6. The paper is about prevention rather than treatment of NOMA

Source: Authors table

\subsection{Data extraction and analysis}

Articles are appraised and data extracted using a structured, pro forma designed a priori from the Cochrane Collaboration's data collection form for intervention reviews, see the table below. This data collection template is pilot tested in randomly selected articles, before being used to assess all included articles. Data extracted includes patient demographics (gender, age, comorbidities), the details of the surgical procedure used (technique, surgery time, intraoperative complications) and any outcome measures reported including time to outcome measurement and any complications (see Appendix 1 ).

\subsection{Methodological Quality and Assessment of Bias}

Article review includes analysis of study design to ascertain risk of bias. Studies are also assigned a level of evidence based on the Oxford Centre for Evidence Based Medicine (10). Included articles are rated for methodological quality using the MINORS criteria as seen in appendix 2 (11).

Table 6- Adapted form of Oxford Levels of Evidence 


\begin{tabular}{|ll|}
\hline $\begin{array}{l}\text { Level of } \\
\text { Evidence }\end{array}$ & Types of Studies \\
\hline I & SR (with homogeneity) of RCTs, Individual RCT (with narrow Confidence Interval), \\
\hline II & $\begin{array}{l}\text { SR (with homogeneity) of cohort studies, Individual cohort study (including low quality RCT; e.g., <80\% follow-up), "Outcomes" Research; } \\
\text { Ecological studie }\end{array}$ \\
\hline III & SR (with homogeneity*) of case-control studies, Individual Case-Control Study \\
\hline V & Case-series (and poor quality cohort and case-control studies) \\
\hline
\end{tabular}

Source: Burns PB, Rohrich RJ, Chung KC. The levels of evidence and their role in evidence-based medicine. Plastic and reconstructive surgery. 2011;128(1):305-10.

\subsection{Statistical analysis}

Data are analysed using IBM SPSS statistics version 24. Descriptive statistics are performed where possible to permit for narrative synthesis. Meta-analysis is performed for outcomes of interest where at least three comparative studies, with low risk of bias, report the same outcome variable.

\section{Abbreviations}

CEBM- Center of evidence-based medicine

IQR- Interquartile range

MINORS- Methodological index for non-randomized studies

MO- Mouth opening

NOITULP- anagram as classification system for NOMA disease

PRISMA- Preferred reporting items for systematic reviews and meta-analyses

WHO- World Health Organization

\section{Declarations}

\section{Ethical Approval and Consent to participate}

Not applicable

\section{Consent for publication}

Not applicable

\section{Availability of supporting data}

Not applicable

\section{Competing interests}

The authors declare that they have no competing interests.

\section{Funding}

This research did not receive any specific grant from funding agencies in the public, commercial, or not-for-profit sectors.

\section{Authors' contributions}

BL, SS and WR designed the study in cooperation. SS and HK planned the original review protocol, which was revised by WR. MB and SS conducted the pilot literature searches, SS built and carried out the final search. SS drafted the manuscript with inputs by BL. WR and HK contributed to manuscript revision. All authors read and approved the final manuscript prior to submission. SS is the primary author and guarantor of this protocol. 


\section{Acknowledgements}

We would like to acknowledge the Nomahilfe E.v. and their great work done in Niger, West Africa and their lead local surgeon Mr. Issa Hamady, whom without these missions and continuing improvement would not be possible.

\section{Authors' information}

${ }^{a}$ Division of Surgery \& Interventional Science, University College London, London, United Kingdom

${ }^{b}$ Charles Wolfson Center for Reconstructive Surgery, Royal Free Hospital, London, United Kingdom

'Department of Plastic Surgery, Royal Free Hospital, London, United Kingdom

${ }^{\mathrm{d}}$ Karl Landsteiner University of Health Sciences, Krems, Austria

\section{References}

1. Bourgeois DM, Leclercq MH. The World Health Organization initiative on noma. Oral Dis. 1999;5(2):172-4.

2. Srour ML, Marck K, Baratti-Mayer D, Noma. Overview of a Neglected Disease and Human Rights Violation. Am J Trop Med Hyg. 2017;96(2):268-74.

3. Chandra RK. 1990 McCollum Award lecture. Nutrition and immunity: lessons from the past and new insights into the future. Am J Clin Nutr. 1991;53(5):1087-101.

4. Leila Srour M, Marck KW, Baratti-Mayer D. Noma: neglected, forgotten and a human rights issue. Int Health. 2015;7(3):149-50.

5. Beisel WR. Nutrition, Function I. Overview The Journal of Nutrition. 1996;126(suppl_10):2611S-5S.

6. Baratti-Mayer D, Gayet-Ageron A, Hugonnet S, Francois P, Pittet-Cuenod B, Huyghe A, et al. Risk factors for noma disease: a 6-year, prospective, matched case-control study in Niger. Lancet Glob Health. 2013;1(2):e87-96.

7. Baratti-Mayer D, Pittet B, Montandon D, Bolivar I, Bornand JE, Hugonnet S, et al. Noma: an "infectious" disease of unknown aetiology. Lancet Infect Dis. 2003;3(7):419-31.

8. Holle J, Kubiena H, Issa OH. Distraction Therapy to Correct Trismus Following Noma. J Craniofac Surg. 2020;31(2):488-91.

9. Moher D, Shamseer L, Clarke M, Ghersi D, Liberati A, Petticrew M, et al. Preferred reporting items for systematic review and meta-analysis protocols (PRISMA-P) 2015 statement. Syst Rev. 2015;4:1.

10. Burns PB, Rohrich RJ, Chung KC. The levels of evidence and their role in evidence-based medicine. Plastic reconstructive surgery. 2011;128(1):305-10.

11. Slim K, Nini E, Forestier D, Kwiatkowski F, Panis Y, Chipponi J. Methodological index for non-randomized studies (minors): development and validation of a new instrument. ANZ J Surg. 2003;73(9):712-6.

\section{Appendix}

\begin{tabular}{|c|c|c|c|c|c|c|c|c|c|c|c|c|c|c|}
\hline \multicolumn{6}{|c|}{ Study Descriptives } & \multicolumn{9}{|c|}{ Demographics and Comorbidities } \\
\hline $\begin{array}{l}\text { Study } \\
\text { No. }\end{array}$ & Authors & Year & Country & $\begin{array}{l}\text { Study } \\
\text { Type }\end{array}$ & $\begin{array}{l}\text { Level of } \\
\text { Evidence }\end{array}$ & $\begin{array}{l}\text { No of } \\
\text { Patients } \\
\text { Included }\end{array}$ & age & sex & BMI & Smoker & Malnutrition & $\begin{array}{l}\text { HIV } \\
\text { positive }\end{array}$ & Measles & Tuberculosis \\
\hline
\end{tabular}

\begin{tabular}{|c|c|c|c|c|c|c|c|c|c|c|c|}
\hline \multicolumn{6}{|l|}{ Surgery } & \multicolumn{6}{|c|}{ Outcomes } \\
\hline $\begin{array}{l}\text { mean no. } \\
\text { of } \\
\text { operations }\end{array}$ & $\begin{array}{l}\text { Distracteur } \\
\text { implantation }\end{array}$ & $\begin{array}{l}\text { Free } \\
\text { microsurgical } \\
\text { flap }\end{array}$ & $\begin{array}{l}\text { Regional } \\
\text { Flap }\end{array}$ & $\begin{array}{l}\text { Local } \\
\text { Flap }\end{array}$ & $\begin{array}{l}\text { Excision } \\
\text { and } \\
\text { direct } \\
\text { closure }\end{array}$ & $\begin{array}{l}\text { follow } \\
\text { up } \\
\text { time }\end{array}$ & $\begin{array}{l}\text { no. of } \\
\text { complications }\end{array}$ & $\begin{array}{l}\text { objective } \\
\text { outcomes- } \\
\text { short term }\end{array}$ & $\begin{array}{l}\text { subjective } \\
\text { outcomes- } \\
\text { short term }\end{array}$ & $\begin{array}{l}\text { objective } \\
\text { outcomes- } \\
\text { long term }\end{array}$ & $\begin{array}{l}\text { subject } \\
\text { outcorr } \\
\text { long te }\end{array}$ \\
\hline
\end{tabular}




\begin{tabular}{|c|c|c|c|c|c|c|c|c|c|c|c|c|c|}
\hline \multicolumn{14}{|c|}{ MINORS Criteria } \\
\hline Study No. & 1 & 2 & 3 & 4 & 5 & 6 & 7 & 8 & 9 & 10 & 11 & 12 & Total \\
\hline
\end{tabular}

Criteria: 1: a clearly stated aim, 2: Inclusion of consecutive patients, 3: prospective collection of data, 4: endpoints appropriate to the aim of the study, 5: Unbiased assessment of the study endpoint, 6: Follow-up period appropriate to the aim of the study, 7: Loss to follow-up less than 5\%, 8: Prospective calculation of the study size, 9: An adequate control group, 10: Contemporary groups, 11: Baseline equivalence of groups, and 12: Adequate statistic analyses. The items are scored 0 (not reported or inadequate) or 1 (adequate). The global ideal score being 16 for non-comparative studies and 24 for comparative sudies

\section{Appendix 2- MINORS CRITERIA}

\section{Table 7- MINORS Criteria}

\section{MINORS criteria}

1. A clearly stated aim: the question addressed should be precise and relevant in the light of available literature

2. Inclusion of consecutive patients: all patients potentially fit for inclusion (satisfying the criteria for inclusion) have been included in the study during the study period (no exclusion or details about the reasons for exclusion)

3. Prospective collection of data: data were collected according to a protocol established before the beginning of the study

4. Endpoints appropriate to the aim of the study: unambiguous explanation of the criteria used to evaluate the main outcome which should be in accordance with the question addressed by the study. Also, the endpoints should be assessed on an intention-to-treat basis.

5. Unbiased assessment of the study endpoint: blind evaluation of objective endpoints and double-blind evaluation of subjective endpoints. Otherwise the reasons for not blinding should be stated

6. Follow-up period appropriate to the aim of the study: the follow-up should be sufficiently long to allow the assessment of the main endpoint and possible adverse events

7. Loss to follow up less than 5\%: all patients should be included in the follow up. Otherwise, the proportion lost to follow up should not exceed the proportion experiencing the major endpoint

8. Prospective calculation of the study size: information of the size of detectable difference of interest with a calculation of $95 \%$ confidence interval, according to the expected incidence of the outcome event, and information about the level for statistical significance and estimates of power when comparing the outcomes

\section{Additional criteria in the case of comparative study}

9. An adequate control group: having a gold standard diagnostic test or therapeutic intervention recognized as the optimal intervention according to the available published data

10. Contemporary groups: control and studied group should be managed during the same time period (no historical comparison)

11. Baseline equivalence of groups: the groups should be similar regarding the criteria other than the studied endpoints. Absence of confounding factors that could bias the interpretation of the results

12. Adequate statistical analyses: whether the statistics were in accordance with the type of study with calculation of confidence intervals or relative risk

Source: Slim K, Nini E, Forestier D, Kwiatkowski F, Panis Y, Chipponi J. Methodological index for non-randomized studies (minors): development and validation of a new instrument. ANZ J Surg. 2003;73(9):712-6.

\section{Figures}




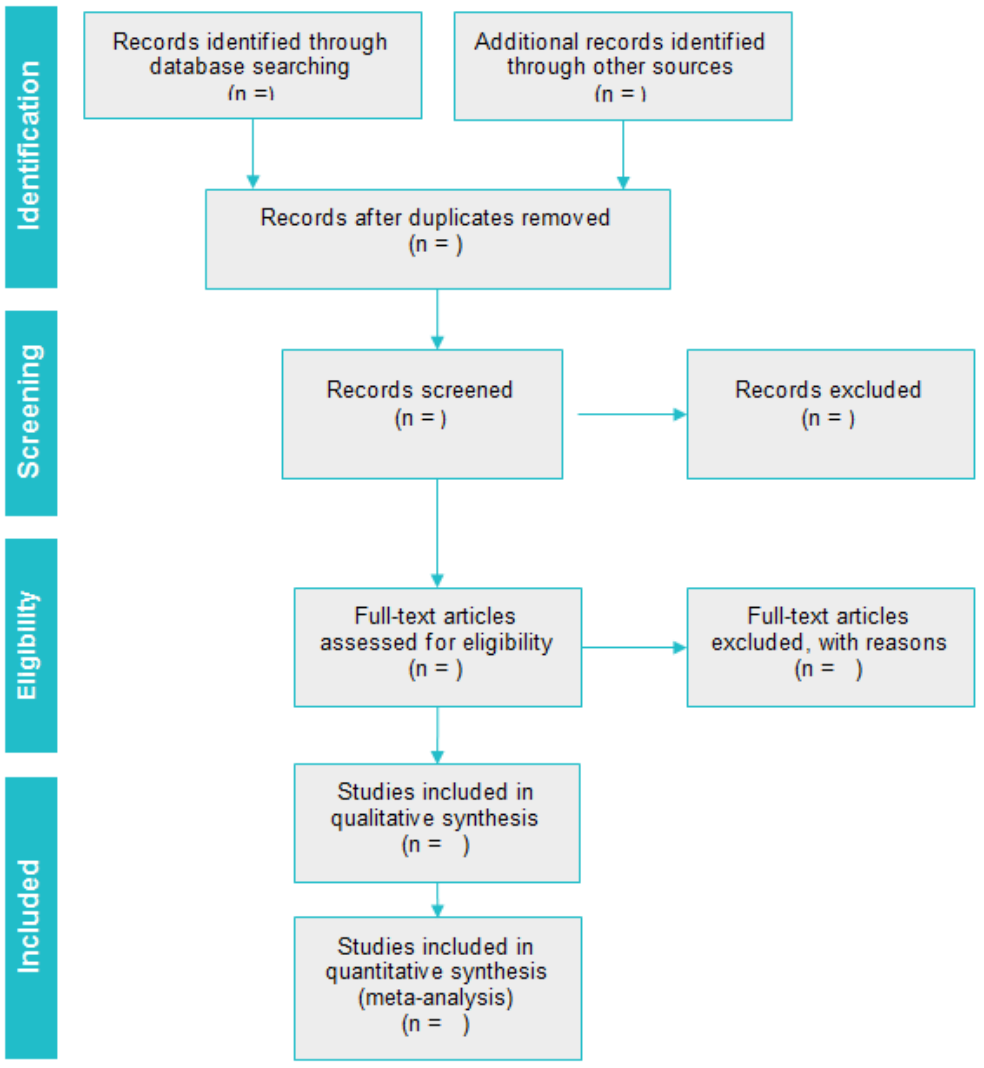

Figure 1

Table 4- PRISMA flow chart for article selection process. Source: Moher D, Shamseer L, Clarke M, Ghersi D, Liberati A, Petticrew M, et al. Preferred reporting items for systematic review and meta-analysis protocols (PRISMA-P) 2015 statement. Syst Rev. 2015;4:1.

\section{Supplementary Files}

This is a list of supplementary files associated with this preprint. Click to download.

- PRISMAPchecklistNOMA.docx 